\title{
Elementary dispersion analysis of some mimetic discretizations on triangular C-grids
}

\author{
P. Korn ${ }^{1}$, S. Danilover,3 \\ ${ }^{1}$ Max Planck Institute for Meteorology, Hamburg, Germany \\ ${ }^{2}$ Alfred Wegener Institute for Polar and Marine Research, Bremerhaven, \\ Germany \\ ${ }^{3}$ A. M. Obukhov Institute of Atmospheric Physics, Moscow, Russia
}

\begin{abstract}
Spurious modes supported by triangular C-grids limit their application for modelling large-scale atmospheric and oceanic flows. Their behavior can be modified within a mimetic approach that generalizes the scalar product underlying the triangular C-grid discretization. The mimetic approach provides a discrete continuity equation which operates on an averaged combination of normal edge velocities instead of normal edge velocities proper. An elementary analysis of the wave dispersion of the new discretization for Poincaré, Rossby and Kelvin waves shows that, although spurious Poincaré modes are preserved, their frequency tends to zero in the limit of small wavenumbers, which removes the divergence noise in this limit. However, the frequencies of spurious and physical modes become close on shorter scales indicating that spurious modes can be excited unless high-frequency short-scale motions are effectively filtered in numerical codes. We argue that filtering by viscuous dissipation is more efficient in the mimetic approach than in the standard C-grid discretization. Lumping of mass matrices appearing with the velocity time derivative in the mimetic discretization only slightly reduces the accuracy of the wave dispersion and can be used in practice. Thus, the mimetic approach cures some difficulties of the traditional triangular C-grid discretization but may still need appropriately tuned viscosity to filter small scales and high frequencies in solutions of full primitive equations when these are excited by nonlinear dynamics.
\end{abstract}

Keywords: 


\section{Introduction}

Most of today's ocean models are formulated on quadrilateral grids with an Arakawa C-staggering that locates pressure points on cell centers and normal components of the velocity vector at cell edges. The reason for the popularity of C-grids in computational geophysical fluid dynamics is the compact stencil for computing pressure gradients and divergence resulting in accurate numerical dispersion relations for waves governed by dynamical equations. This placement of the variables within the quadrilateral grid cells is free of pressure modes that exist on the Arakawa A- and B-grids because of pressure gradient averaging. Moreover, following [1], a C-grid discretization can be made mimetic such that the discrete operators of divergence, curl and gradient have the properties of their continuous analogs.

The properties mentioned above explain why a C-grid staggering seem to be a natural choice for the discretization of the equation of geophysical fluid dynamics on unstructured meshes such as triangular or even general polygonal grids [13]. Indeed, in a number of works it was shown that the triangular Cgrid discretization, and its finite-element counterpart, the $\mathrm{RT}_{0}-\mathrm{P}_{0}$ discretization [12] ensures an accurate representation of propagating long surface waves (see, e. g., [10]) and allows the geostrophic balance to be maintained at a local level [14]. The $\mathrm{RT}_{0}-\mathrm{P}_{0}$ discretization is more flexible and can be formulated on arbitrary triangular meshes, its relation to the standard triangular C-grid is discussed in [16]. As a consequence, triangular C-grids are the discretization chosen by some regional coastal and estuarine ocean models such as UnTRIM [3], ELCIRC [2] or SUNTANS [5], where the accuracy of wave dynamics is of primary importance. Triangular C-grid has also been proposed by Stuhne and Peltier [15] as the basis of a large-scale ocean model formulated in a spherical geometry.

For global ocean- or atmosphere models, it gradually has become clear that triangular C-grids exhibit noise in the divergence of horizontal velocity and hence in the vertical velocity. The origin of this noise is purely geometrical and relates to the imbalance in the numbers of the degrees of freedom available on triangular C-grids for velocity and pressure. Since on large triangular meshes the ratio of the number of edges to that of cells is 3 to 2 , there are too many pressure degrees of freedom. As a result, there are spurious inertiagravity modes; the field of horizontal velocity divergence associated to them corresponds to a triangular checkerboard pattern. In [10] it is demonstrated that this noise becomes noticeable if the Rossby radius of deformation is not 
resolved. The origin of this noise is also discussed in [6] and [17], and some of its implications are illustrated in [4]. The practical usage of triangular Cgrids for large-scale simulations relies, therefore, on filtering [18], divergence averaging [19] or special dissipation that suppresses the component of the horizontal velocity field which might project on the divergence noise [17].

However, a generalization of the classical C-grid discretization is possible, as proposed in [7], which relies on a different form of scalar product than implied by traditional C-grids. It leads to a mimetic discretization which conserves energy and enstrophy [8]. In essence, it introduces a new velocity, linked to the edge velocity through a certain averaging operator. This new velocity, and not the edge velocity, satisfies the continuity equation. It turns out that this generalization leads to dramatic improvements, being much less vulnerable to spurious noise. Numerical simulations that support this statement can be found in [7].

The purpose of this paper is to present an elementary analysis of dispersion properties of linearized shallow water equations discretized with this new generalized approach on a triangular mesh. We show how the spurious modes are modified and why their manifestations become much less damaging while the conservation properties are stillmaintained. We will also explore the effects of lumping of the "mass matrices" that appear in this approach. These matrices appear in the time derivative of the velocity equations, similar to continuous Finite Element methods, and therefore we refer to them as "mass matrices" and to the simplification of this marices as "lumping. We note that in our approach these matrices occur as consequence of reconstruction operators in the energy norm rather than from basis expansions as in Finite Element methods. Lumping the mass matrix improves the computational efficiency, it formally destroys energy conservation (but not mass conservation, see also [7]) but our analysis shows that it affects the accuracy of simulated waves only slightly. We point out that in the limit of linear shallow-water equations the lumped configurations are very similar to the above mentioned technique of divergence averaging. The notable advantage of mimetic discretization is that it stays consistent even on non-uniform meshes. An analysis of similar approaches on quadrilateral cells reveals that the effect of lumping might differ on different geometries (see Appendix B).

We start from discussing the reconstruction operators and energy norms associated to discretizations of C-grid type. We then turn to the Poincaré waves, considering the case of constant Coriolis parameter and doing the standard von Neumann analysis. The analysis of the Rossby and Kelvin waves will be 
done numerically. As a step aside we illustrate the application of generalized approach to quadrilateral meshes where analytical computations are more straightforward.

\section{Discrete Framework}

\subsection{Equations, Energy Norms and Scalar Products}

The dynamical equations underlying our analysis are the linearized shallow water equations with a flat-bottom

$$
\begin{gathered}
\partial_{t} \mathbf{u}+f \mathbf{k} \times \mathbf{u}+g \nabla \eta=0, \\
\partial_{t} \eta+H \nabla \cdot \mathbf{u}=0,
\end{gathered}
$$

where we use the standard notation, $\mathbf{u}$ denotes the two-dimensional velocity vector, $\eta$ the fluid thickness and $H$ the fluid depth, $g$ is the gravitational constant. We either assume an f-plane, $f=f_{0}$ or a $\beta$-plane, $f=f_{0}+\beta y$. These equations are further discretized using the C-grid variable placement. We introduce the edge normal velocities $v_{e}$ located at mid-edges, and cell elevations $\eta_{c}$ located on the cell circumcenters. The indices $e$ and $c$ label the edges and cells respectively. This variable placement allows for several possibilities of discretization. The first one is the standard C-grid, the other is the finite-element $R T_{0}-P_{0}$ discretization, and still other follow from the mimetic approach and will be detailed further. We will briefly address them further to stress the differences.

The essential difference of different discretizations of the shallow water equations (1), (2) is how they define the energy norm. The standard C-grid assumes the following energy norm

$$
2 E=2 K+2 P=H \sum_{e} l_{e} d_{e} v_{e}^{2}+g \sum_{c} A_{c} \eta_{c}^{2}
$$

which is the combination of two scalar products for kinetic and potential energy,

$$
S_{k i n}(\mathbf{a}, \mathbf{b}):=\sum_{e} l_{e} d_{e} a_{e} b_{e}
$$

and

$$
S_{p o t}(a, b):=\sum_{c} A_{c} a_{c} b_{c}
$$


In the expressions above $K$ and $P$ are the kinetic and potential energies respectively. We use the notation $l_{e}, d_{e}$ and $A_{c}$ for the edge length, the distance between the circumcenters across the edge and triangle area respectively. The presence of scalar products and the consistency between them for the selected discrete divergence and gradient operators is essential for ensuring that the discretization is energy conserving. The standard triangular C-grid assumes the following divergence operator

$$
D_{c e} v_{e}=-A_{c}^{-1} \sum_{e} l_{e} v_{e} \delta_{e c},
$$

where $\delta_{e c}$ is 1 for inner normals, - 1 for outer, or 0 if $e$ and $c$ are not related. With this divergence operator

$$
g H S_{p o t}(\eta, \nabla \cdot \mathbf{u})=-g H \sum_{c} \eta_{c} \sum_{e} l_{e} v_{e} \delta_{e c}=-g H S_{k i n}(\mathbf{u}, \nabla \eta)
$$

for the most natural gradient operator

$$
G_{e c} \eta_{c}=\sum_{c} \eta_{c} \delta_{e c} / d_{e}
$$

For both operators $(D$ and $G$ ) summation over repeated indices is implied in matrix-vector products. These expressions for the divergence and gradient operators naturally arise in the standard C-grid discretization. They ensure that the transfer of potential to kinetic energy (the rhs of (3)) is opposite to the transfer of kinetic to the potential energy (the lhs of (3)). The Coriolis term requires a reconstruction that ensures an antisymmetric operator in the energy norm, which is done with the help of Perot reconstruction (see below). Clearly, to achieve conservation in the nonlinear case the scalar products have to be modified to include the full layer thickness, and the Perot reconstruction have to be reconsidered too (see [9]).

In the case of $R T_{0}-P_{0}$ finite-element discretization the test-functions used to represent velocity on triangles are vector-valued. The scalar product in this case is introduced by the Galerkin discretization

$$
S_{k i n}(\mathbf{a}, \mathbf{b})=\int_{c} \mathbf{a}_{c} \cdot \mathbf{b}_{c} d S_{c}
$$

where the vectors belong to the space of discrete velocities, and $S_{p o t}$ is left without changes. The velocity $\mathbf{u}_{c}$ on triangle $c$ is expanded in basis functions 
$\mathbf{u}_{c}=-\sum_{e} v_{e} \boldsymbol{\psi}_{e c} \delta_{e c}$ where $\boldsymbol{\psi}_{e c}=\left(\mathbf{r}-\mathbf{r}_{e c}\right) / h_{e c}$, with $\mathbf{r}$ and $\mathbf{r}_{e c}$, respectively, the radius-vector and the radius-vector drawn to the vertex opposing edge $e$ in triangle $c$, and $h_{e c}$ the height drawn to the edge from the vertex. Computation of $\nabla \mathbf{u}_{c}$ leads to the same expression for the divergence operator as on C-grid. Since the representation of elevation is elementwise constant, one uses the weak definition of the gradient, obtained by integration by parts

$$
\int \tilde{\boldsymbol{\psi}}_{e c} \nabla \eta d S=-\int \nabla \cdot \tilde{\boldsymbol{\psi}}_{e c} \eta d S
$$

where the contributions from the edges are set to zero since the normal component of test function $\tilde{\boldsymbol{\psi}}_{e c}$ is continuous. This just defines the gradient as the negative adjoint of the divergence, hence the energetic consistency

$$
S_{k i n}(\mathbf{a}, \nabla \eta)=-S_{p o t}(\nabla \cdot \mathbf{a}, \eta)
$$

follows for any a from the velocity space.

Note that the $R T_{0}-P_{0}$ finite-element discretization easily handles the Coriolis operator because $S_{k i n}$ operates on full vectors. Elementary computations show that the expression for the divergence operator $D_{c e}$ remains the same as for the C-grid above. The gradient operator, appearing in the momentum equation (in area-weighted form), lacks the division over $d_{e}$ which one expects to see. Instead, the time derivative of velocities acquires the mass matrix of the standard Galerkin procedure (given by the scalar product $S_{k i n}$ of the test functions $\boldsymbol{\psi}_{e c}$ ). The aspect of 'distance' (between cell centers) is set by the mass matrix if it is lumped. There are two common ways of lumping, one leading to the representation analogous to $\mathrm{C}$-grids and requiring orthogonal meshes, where the lines connecting cell centers and vertices are perpendicular, and the other one based on the distance between centroids of cells on both sides of edge $e$, (see e. g. [16]). The latter case and the case with the consistent mass matrix formally work on general triangular meshes.

\subsection{Mimetic Variants on $C$-grids}

A much wider set of possibilities is opened by replacing the scalar product (4) by the following one

$$
S_{k i n}(\mathbf{a}, \mathbf{b})=\sum_{c} A_{c} \mathbf{P}_{c e} a_{e} \cdot \mathbf{P}_{c e^{\prime}} b_{e^{\prime}}
$$

where $P_{c e}$ is an appropriate reconstruction operator that takes normal components of vectors and reconstructs full vectors at cell centers. Through (5) 
one implements a weighted scalar product. The Perot operators that we consider here represent one possible choice for the reconstruction on cell centers and on vertices. The forward Perot reconstruction operator [11] is given by

$$
P v_{e}:=A_{c}^{-1} \sum_{e} v_{e} l_{e}\left(\mathbf{x}_{c}-\mathbf{x}_{e}\right) \delta_{e c}
$$

Here $\mathbf{x}_{c}$ and $\mathbf{x}_{e}$ are the radius-vectors drawn to the circumcenter of cell $c$ and mid-edge of edge $e$. The backward Perot operator maps vectors at cell circumcenters to scalar variables at edges,

$$
P^{T} \mathbf{u}_{c}:=d_{e}^{-1} \sum_{c} \mathbf{u}_{c}\left(\mathbf{x}_{c}-\mathbf{x}_{e}\right) \delta_{e c}
$$

The operator $P^{T}$ is transpose of $P$ and is its adjoint in the sense of scalar product defined above. We note that $P$ returns a vector from scalars, and $P^{T}$ acts on vector and returns a scalar, but we will use a simplified notation that does not explicitly indicate it.

Additionally, we introduce the Perot vertex reconstruction operator, that reconstructs a vector at the triangles vertices from normal components at edges. The vertices are the cell centers of the dual grid, consisting of hexagons, that are created by connecting the triangular cell centers around a vertex. The Perot vertex reconstruction operator is defined as

$$
\hat{P} v_{e}:=A_{v}^{-1} \sum_{e} v_{e} d_{e}\left(\mathbf{k} \times \mathbf{x}_{v}-\mathbf{x}_{e}^{*}\right) \delta_{e v}
$$

Its derivation follows the same logic as for $P$, but dual cells are considered instead of triangles, so that $\mathbf{x}_{e}^{*}$ are the mid-centers of dual edges, and $\delta_{e v}$ is defined similarly to $\delta_{e c}$. To be consistent $\delta_{e v}=1$ if edge $e$ is oriented to $v$ and $\delta_{e c}=1$ for the right triangle $c,-1$ for the other sign of $\delta_{e c}=1$, and 0 if $e$ does not contain $v$. The backward vertex reconstruction operator maps a vector at vertices to a tangential component at mid-edges and is defined by

$$
\hat{P}^{\dagger} \mathbf{a}_{v}:=l_{e}^{-1} \sum_{v} \mathbf{a} \cdot\left(\mathbf{x}_{v}-\mathbf{x}_{e}^{*}\right) \delta_{e v}
$$

The Perot operators on the primal grid create as product $P^{T} P$ a symmetric and positive definite operator that defines a scalar product, while the dual grid product $\hat{P}^{\dagger} \hat{P}$ established a skew-symmetric operator. 
This road leads to a mimetic discretization. Using the Perot operator and its transposed one can express the kinetic energy, defined in (5) as a cell quantity, as a sum over edges

$$
S_{k i n}(\mathbf{a}, \mathbf{b})=\sum_{e} l_{e} d_{e} b_{e}\left(P^{T} P\right)_{e e^{\prime}} a_{e^{\prime}}=\sum_{e} l_{e} d_{e} a_{e}\left(P^{T} P\right)_{e e^{\prime}} b_{e^{\prime}}
$$

From this equation one can derive the discrete momentum equation related to the selected scalar product by substituting for $\mathbf{b}$ the time derivative or pressure gradient in (1). The gradient operator becomes then $G_{M}=P^{T} P G$ with $G$ defined above for the C-grid and accordingly is the energetically consistent divergence operator now given by $D_{M}=D P^{T} P$. The admissible reconstruction operators should possess the property that $P^{T} P$ produces identical result acting on a uniform velocity field. Note that, different from the two other cases above, here the continuity is satisfied by the velocity field $P^{T} P v_{e}$, and not by the field $v_{e} . P^{T} P$ is thus a filter operator, and $P^{T} P v_{e}$ is the physical (observable) field while $v_{e}$ is just a seed. It will be demonstrated that filters filters the velocity component that projects on the divergence noise in the limit of small wavenumbers.

Energy neutrality is required from the Coriolis operator and we consider two possibilities to represent it. The first one relies on the reconstruction $\mathbf{u}_{c}=P v_{e}$ of the velocity vector to cell circumcenters, computing the Coriolis acceleration $f \mathbf{k} \times \mathbf{u}_{c}=f \mathbf{k} \times P v_{e}$ there, and projecting this acceleration back to edges (see e.g. SUNTANS and [15]). This defines the Coriolis operator as $C v_{e}=P^{T}\left(f \mathbf{k} \times P v_{e}\right)$, which is also obtained in $R T_{0}-P_{0}$ finite-element discretization. The second possibility is to approximate the Coriolis operator as $C_{M} v_{e}=\hat{P}^{\dagger}\left(f \hat{P} v_{e}\right)$, using the forward and backward Perot operators on vertices. Thus, the dual grid consisting of hexagons is involved in the discretization of the Coriolis operator, while the Coriolis discretization $C$ uses only the primal grid, given by triangles. The advantage of this approach is that it not only allows energy conservation, as the first Coriolis discretization above, but also potential enstrophy conservation [8]. The null space of $C_{M}$ as well as of $C$ consist of the zero only [7].

Since the finite-element $R T_{0}-P_{0}$ discretization shares the operator of divergence with the standard C-grid, it is not different from the $\mathrm{C}$-grid in what concerns the behavior of spurious modes, and will not be considered further. We compare, therefore, the standard C-grid discretization to several variants of implementing the mimetic discretization.

With the notation for operators introduced above, the standard C-grid dis- 
cretization becomes

$$
\begin{array}{ll}
\text { Standard-C: } \quad & \partial_{t} v_{e}+C v_{e}+g G \eta_{c}=0, \\
& \partial_{t} \eta_{c}+H D v_{e}=0 .
\end{array}
$$

The mimetic discretization proposed in [7] corresponds to

$$
\begin{array}{ll}
\text { Mimetic-Dual: } & \partial_{t} P^{T} P v_{e}+C_{M} v_{e}+g P^{T} P G \eta_{c}=0, \\
& \partial_{t} \eta_{c}+H D P^{T} P v_{e}=0
\end{array}
$$

The same discretization, with $C_{M}$ replaced by $C$ reads as follows

$$
\begin{array}{ll}
\text { Mimetic-Primal: } & \partial_{t} P^{T} P v_{e}+C v_{e}+g P^{T} P G \eta_{c}=0, \\
& \partial_{t} \eta_{c}+H D P^{T} P v_{e}=0 .
\end{array}
$$

Both mimetic approaches above contains the mass matrix $\left(P^{T} P\right)$ for velocity. The inversion of mass matrix will slow down the performance, so of interest are the variants where it is lumped (replaced with the identity matrix), and we consider two possibilities,

$$
\begin{array}{ll}
\text { Mimetic-Lumped-1: } \quad & \partial_{t} v_{e}+C_{M} v_{e}+g G \eta_{c}=0 \\
& \partial_{t} \eta_{c}+H D P^{T} P v_{e}=0 .
\end{array}
$$

and

$$
\begin{array}{ll}
\text { Mimetic-Lumped-2: } & \partial_{t} v_{e}+C_{M} v_{e}+g P^{T} P G \eta_{c}=0, \\
& \partial_{t} \eta_{c}+H D P^{T} P v_{e}=0 .
\end{array}
$$

The latter variant introduces a larger error in the momentum equation, but keeps, in contrast to Mimetic-Lumped-1, the symmetry between the gradient and divergence.

The equations proposed above satisfy different discrete conservation laws. All variants conserve mass, the standard C-grid and the mimetic primal both conserves total energy, while the mimetic dual conserves energy and enstrophy [8], and the two lumped configurations conserve neither energy nor enstrophy. 


\section{Analysis of wave dispersion}

\subsection{Poincaré waves}

We seek for solutions of discretized equations (6) -(10) varying as $\exp (-i \omega t+$ $i k x+i l y)$ and assume an f-plane approximation and an infinite (spatial) domain. In a continuous case the solution includes inertia-gravity waves, referred to as the Poincaré waves, and a stationary geostrophic mode.

Consider an infinite mesh formed of equilateral triangles with a side $a$ and the height $h=a \sqrt{3} / 2$. Introduce a Cartesian coordinate system $(x, y)$ and two generating triangles: one pointing northward (upward) with vertices at the points $(-a / 2,0),(a / 2,0)$ and $(0, h)$ and the other one pointing southward (downward) with the coordinates $(-a / 2,0),(a / 2,0)$ and $(0,-h)$. All other mesh triangles are obtained by translations from these two. The subscripts $u$ and $d$ will be used to label quantities related to such triangles. To describe our discretization we need to introduce 5 degrees of freedom: the elevations on $\mathrm{u}$ - and d-triangles and velocities on three sides of triangles, i.e., our state vector is $\left(V_{a}, V_{b}, V_{c}, E_{u}, E_{d}\right)=\left(v_{a}, v_{b}, v_{c}, \eta_{u}, \eta_{d}\right) e^{-i \omega t+i k x+i l y}$. For a $u$-triangle, we associate $V_{a}$ with the side oriented zonally, $V_{b}$ with the edge emanating from $(-a / 2,0)$ and $V_{c}$ with the edge emanating from $(a / 2,0)$. Additionally, we assume that normals are inner for $u$-triangles. Although the phase is defined relative $(0,0)$, any other point can be taken in computations for only relative phases will enter the relationships between quantities. Below all operators will be written relative the points where they are defined. One can readily see that all discrete degrees of freedom are obtained from these five by translations, and can be taken into account through phase multipliers. Analogously, the operators appearing in the equations can be reduced to a $3 \times 3$ matrix for $P^{T} P$ and the Coriolis operators $C$ and $C_{M}$, to a 3 $\times 2$ matrix for the gradient $G$ and to a $2 \times 3$ matrix for the divergence $D$. These matrices are functions of wavenumbers. Deriving them is straightforward. In order to let all matrices act on the full state vector we augment

$C, C_{M}, G$ and $D$ to $5 \times 5$ matrices operating on the full state vector by filling the added parts with zeros. Furthermore we introduce the mass matrix $T$ with $T(1: 3,1: 3)=P^{T} P, T(4,4)=T(5,5)=1$ and remaining values are zeros. The same notation is kept for original and augmented matrices, as it is always clear from the context what is meant. With these conventions we formulate the eigenvalue problems for $5 \times 5$ matrices corresponding to the different discretizations. 
For the standard C-grid (6) we obtain

$$
\text { Standard-C: } \quad M_{C}:=C+g G+H D .
$$

For the case of Mimetic-Dual (7) we have

$$
\text { Mimetic-Dual: } \quad M_{\text {Dual }}=T^{-1}\left(C_{M}+g G_{M}+H D_{M}\right),
$$

where $G_{M}:=P^{T} P G$ and $D_{M}:=D P^{T} P$ are also augmented to the $5 \times 5$ format. For the Mimetic-Primal discretization (8) which differs from MimeticDual by the implementation of the Coriolis operator we get

$$
\text { Mimetic-Primal: } \quad M_{\text {Primal }}=T^{-1}\left(C+g G_{M}+H D_{M}\right) .
$$

The Lumped-1 configuration (9) is described by the matrix

$$
\text { Lumped-1: } \quad M_{L_{1}}=C_{M}+g G+H D_{M} \text {. }
$$

The matrix for the Lumped-2 case (10) reads as follows

$$
\text { Lumped-2: } \quad M_{L_{2}}=C_{M}+g G_{M}+H D_{M} \text {. }
$$

Before analysing these discretizations, an elementary consideration of the discrete divergence operator and its mimetic modification is worthwhile. The $\mathrm{C}$-grid divergence is written as

$$
\begin{aligned}
D_{u}\left(u_{a}, u_{b}, u_{c}\right)^{T}= & (2 / h)\left(-u_{a} e^{-i l h / 3}-u_{b} e^{i k a / 4+i l h / 6}\right. \\
& -u_{c} e^{-i k a / 4+i l h / 6}=(2 / h)\left(-\alpha u_{a}-\beta u_{b}-\gamma u_{c}\right), \\
D_{d}:=-D_{u}^{*}, &
\end{aligned}
$$

where the asterisk implies complex conjugation and we introduced the notation for phase multipliers $\alpha=e^{-i l h / 3}, \beta=e^{i k a / 4+i l h / 6}$ and $\gamma=e^{-i k a / 4+i l h / 6}$. One can form the combinations

$$
\bar{D}:=\left(D_{u}+D_{d}\right) / 2, \quad \tilde{D}:=\left(D_{u}-D_{d}\right) / 2,
$$

such that $D_{u}=\bar{D}+\tilde{D}$ and $D_{d}=\bar{D}-\tilde{D}$. Obviously, $\bar{D}$ has purely imaginary coefficients, and in the limit of small wavenumbers corresponds to the continuous operator of divergence. The other combination, $\tilde{D}$ has real-valued 
coefficients. Its presence leads to known difficulties of the standard C-grid discretization. A Taylor expansion yields for small wavenumbers

$$
\begin{aligned}
(h / 2) \tilde{D}\left(u_{a}, u_{b}, u_{c}\right)^{T}= & \left(u_{a}+u_{b}+u_{c}\right)+(1 / 2)\left[(l h / 3)^{2} u_{a}\right. \\
& \left.+(k a / 4+l h / 6)^{2} u_{b}+(k a / 4-l h / 6)^{2} u_{c}\right]+\ldots
\end{aligned}
$$

Although the vector consistency requires $u_{a}+u_{b}+u_{c}=0$ such that the first term vanishes (see the discussion in [6]), discrete dynamics would not necessarily preserve this condition. This immediately leads to a bias in the divergence reflected in a triangular checkerboard pattern. Even if the property $u_{a}+u_{b}+u_{c}=0$ is respected, the presence of the second term (which corresponds to a second order operator in the Taylor expansion, (cf. [17])) implies only the first order convergence for the divergence operator. The eigenvectors of the matrices occurring in the different discretizations (eqs. (11)-(15)) do not respect the requirement $u_{a}+u_{b}+u_{c}=0$, so the problem can be present for any of them, but its extent depends on the discretization type as will be shown further.

We consider now the "filtered divergence" $D P^{T} P$. An elementary calculation shows that the operator $P^{T} P$ can be expressed as

$$
P^{T} P=\frac{1}{3}\left(\begin{array}{ccc}
2 & -\left(\alpha^{*} \beta / 2+\text { c.c. }\right) & -\left(\alpha^{*} \gamma / 2+\text { c.c. }\right) \\
-\left(\alpha^{*} \beta / 2+\text { c.c. }\right) & 2 & -\left(\beta^{*} \gamma / 2+\text { c.c. }\right) \\
-\left(\alpha^{*} \gamma / 2+\text { c.c. }\right) & -\left(\beta^{*} \gamma / 2+\text { c.c. }\right) & 2
\end{array}\right)
$$

Note that $P$ is in this case the combination $P=\left(P_{x}^{u}, P_{y}^{u}, P_{x}^{d}, P_{y}^{d}\right)$ each of which act on $\left(u_{a}, u_{b}, u_{c}\right)^{T}$. The operator $P^{T}$ is therefore represented by 3 by 4 matrix acting on $x$ and $y$-components of $u$ and $d$ velocities. In the limit of infinitely small wavenumbers the three eigenvalues of $P^{T} P$ are 0 , 1 and 1. The eigenvector related to the first eigenvalue is $(1,1,1)$, i. e., the combination $u_{a}+u_{b}+u_{c}$ is in the null-space of $P^{T} P$. For non-vanishing $k a, l h$ the operator $P^{T} P$ possesses full rank. One of its eigenvalues stays equal 1 , which is obvious geometrically, but the two others are between 0 and 1 and tend to each other as the wavenumbers are increased. For this reason $P^{T} P$ behaves as a smoothing operator. The presence of small eigenvalue at small wavenumbers damps the combination of velocities that may project on divergence noise.

Getting an idea of the effect of $P^{T} P$ is much simpler on quadrilateral meshes which are considered in Appendix B. 
On the uniform mesh considered here one may easily check that

$$
D P^{T} P=\left(\bar{D}_{u}\right),
$$

with

$$
\left.\bar{D}_{u}=\left[3 D_{u}+\left(\alpha^{2}+\beta^{2}+\gamma^{2}\right) D_{d}\right)\right] / 6
$$

and

$$
\left.\bar{D}_{d}=\left[3 D_{d}+\left(\alpha^{2}+\beta^{2}+\gamma^{2}\right)^{*} D_{u}\right)\right] / 6
$$

and this corresponds to the averaged divergence operators described for example in [17]. It thus follows that $P^{T} P$ of mimetic discretization introduces divergence averaging in a consistent way by (i) providing generalization which is valid on non-uniform meshes and (ii), more importantly, providing a consistent gradient operator and energy conservation.

We continue now the analysis of the eigenvalue problems for $5 \times 5$ matrices corresponding to the discretizations (11) - (15). The analytical expression for the eigenfrequencies (i.e. the eigenvalues multiplied with $i$, the imaginary unit) can be in principle derived by solving det $\left|M_{i}-i \omega I\right|=0$ for the system matrices $M_{i}$ given by equations (11)-(15) above. One of the frequencies is always zero (geostrophic mode), leaving biquadratic equations which couples two physical and two spurious modes. We identify the physical mode as the mode with correct behavior at small wavenumbers. The resultant expressions are however, unwieldy and need to be explored numerically. For this reason it is easier to explore the dispersion numerically from the very beginning. Since we expect that the spurious numerical modes may excite divergence noise, we will use the phase of the ratio of elevation on upward and downward pointing triangles as an an indicator. This is motivated by the observation that by virtue of (2), the divergence is equal to $i \omega \eta$ (with $\eta$ equal to $\eta_{u}$ or $\left.\eta_{d}\right)$, so that the phase of the ratio of elevations is equal to that of the ratio of divergences. For a physical mode at small wavenumbers the phase should be close to zero. A checkerboard pattern in the field of elevation and divergence would manifest itself by a phase that is close to $\pm \pi$.

Figures 1 and 2 plot the dispersion curves and phases for two ratios $r:=a / L_{R}$ between the triangle side $a$ and the Rossby Radius $L_{R}=(g H)^{1 / 2} / f$. Figure 1 corresponds to $r=0.5$ when the Rossby radius is marginally resolved, and Fig. 2 corresponds to a coarse mesh $r=2.5$, for waves propagating at an angle $\pi / 6$. While the numerically simulated dispersion curves depend on the 
direction, their behavior remains very similar. The modes are sorted so that frequency increases with mode number.

The left panels in Fig. 1 and 2 show the dispersion curves for the standard Cgrid (the top panels) and the full mimetic discretization (the bottom panel). The color is blue for the physical Poincaré waves, green for the geostrophic mode and red for spurious modes. The black lines plot the theoretical prediction, $(\omega / f)^{2}=1+r^{-2}\left(k^{2}+l^{2}\right) a^{2}$. Note that spurious and physical modes nearly coalesce in Fig. 1 for the mimetic discretization at $k a>1$, so that the apparent color indicates the order the curves were plotted. On fine meshes, the mimetic discretization is slightly less accurate than the C-grid, which is an expected result of averaging, yet it is still more accurate than many other discretizations (as can be seen from the results of e.g. [10]). The right panels show that spurious modes are present in both cases. They are modes 1 and 5 for C-grid (6) and 2 and 4 for the Mimetic-Dual (7). The phase of the elevation ratio is close to $-\pi$ for these modes indicating that their field of divergence contains a checkerboard pattern (the $u$ - and $d$ - values are opposite). For the C-grid discretization, the frequency of spurious modes is much higher than the frequency of physical modes at small wavenumbers if the Rossby radius is well resolved. As a result, they would not necessarily be excited by slow dynamics. Their frequencies become closer on coarse meshes (compare the top left panels in Fig. 1 and 2), which is by all probability the reason why dynamics become more vulnerable to the excitation of divergence noise in this case. Note that the frequencies of spurious and physical modes approach each other as $k a$ is increased, with the implication that spurious modes may be easier excited by dynamics at small scales.

In the case of Mimetic-Dual discretization (7) the frequency of spurious modes equals zero in the limit of $k, l=0$. This is the manifestation of the effect of $P^{T} P$ in this limit: if a spurious mode is present in $\eta$, it is compatible with the thickness equation only for zero frequency. The reduction in frequency of spurious modes for small wavenumbers is therefore an indicator of the efficiency of suppressing the manifestations of these modes in the divergence field by the mimetic discretization. Put differently, spurious divergence is of much smaller amplitude at small wavenumbers for a given amplitude of interface displacement, and this is why the mimetic discretization remains less prone to the existence of spurious modes. The drawback of Mimetic-Dual discretization is that on coarse meshes it predicts a negative group velocity for the Poincaré waves, i. e., the incorrect propagation 
direction of wave energy (see Fig. 2).
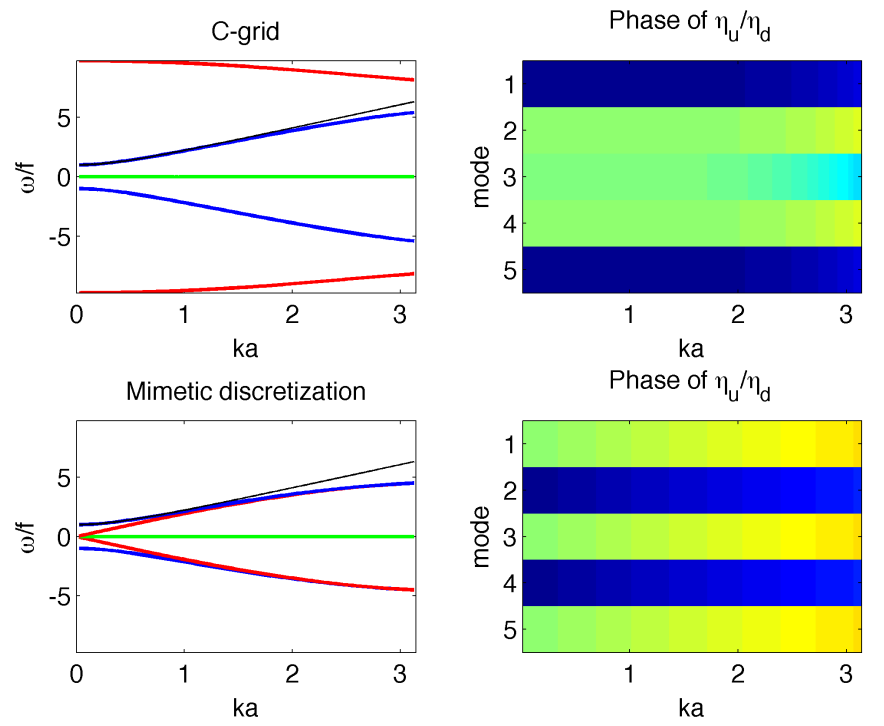

Figure 1: Poincaré waves on a fine mesh $\left(a / L_{R}=1 / 2\right.$, the Rossby radius is resolved). Left: The dispersion of discrete modes for C-grid (top) and mimetic-dual (bottom). The blue lines are physical Poincaré modes, the red lines are spurious modes and the green line is the geostrophic mode. The theoretical dependence is plotted with the black line for $\omega>0$. Right: The phase of the ratio of elevation on $u$ - and $d$-triangles for the eigenvectors of discrete modes sorted in the ascending order by their frequency. The colorbar is from $-\pi$ to $\pi$, and the blue color indicates the presence of a checkerboard pattern (change of sign) in the elevation and, hence, the divergence. Spurious modes are present in both cases, the difference is their frequency, which is low for mimetic at small wavenumbers.

Figures 3 and 4 illustrate the impact of different approximations in the implementation of mimetic discretization on the dispersion characteristics for $a / L_{R}=0.5$ (fine mesh) and 2.5 (coarse mesh) respectively. On fine meshes (Fig. 3), they all perform similarly, although the case Lumped-2 (10) with the lumped mass matrix but consistent gradient shows substantially lower accuracy. There is very little difference between the Mimetic-Dual (7) and its lumped version (9), which confirms that lumping does not deteriorate the accuracy in practice. Likewise, there is very little difference between Mimetic-Dual and Mimetic-Primal, showing that the difference between the two forms of the Coriolis operator is of little relevance for the accuracy of Poincaré wave dispersion on fine meshes. The loss of accuracy of Lumped 2 

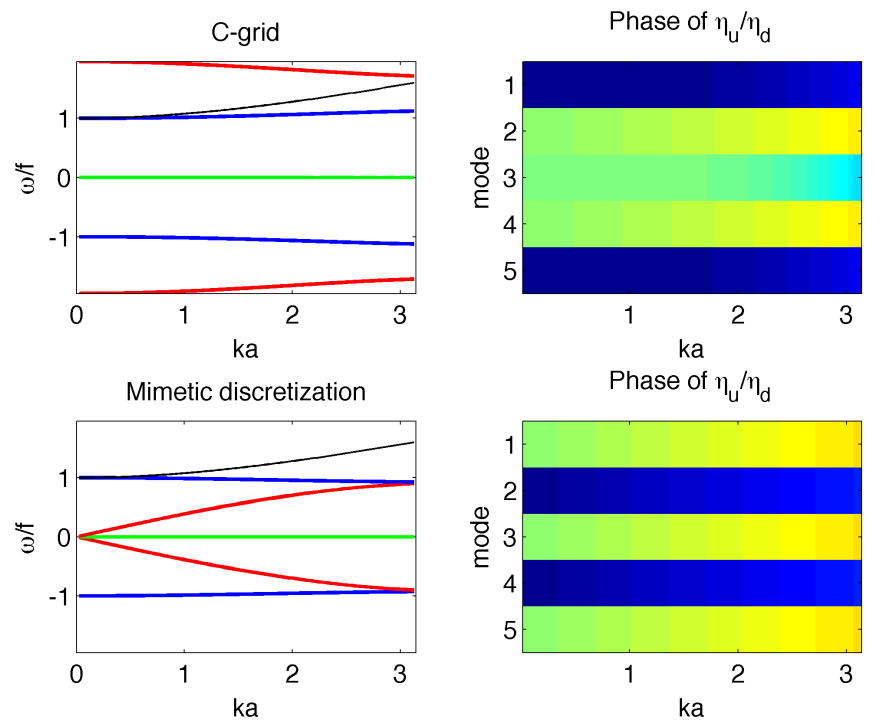

Figure 2: The same as in Fig. 1, but for $a / L_{R}=2.5$ (coarse mesh).

case is also seen for the coarse mesh (Fig. 4), and its group velocity is more negative (compared to full mimetic and Lumped 1). Rather surprisingly the Mimetic-Primal shows the best performance in this case, demonstrating the most accurate behaviour (even with respect to $\mathrm{C}$-grid), with the correct sign of group velocity. Note that some detail of the dependencies shown here may vary with the wave vector direction and the ratio $a / L_{R}$.

The frequency of spurious modes in all variants of mimetic discretization is small at small wavenumbers, so that mimetic discretizations are less vulnerable to the manifestations of spurious modes compared to the $\mathrm{C}$-grid in this limit. Before we investigate in the next section if this property has implications for the Kelvin and Rossby waves because their dispersion curves are close in this case to those of spurious modes, we will study the behaviour of the method in the limit of small wavenumbers and the controllability of the spurious modes by viscous dissipation.

Consider a limit of wavenumbers $k a$, la going to zero. In this case we have $u_{a}, u_{b}, u_{c}$ and $\eta_{u}$ and $\eta_{d}$ as previously, but their dependence on the horizontal coordinate can be ignored. They are only functions of time $e^{-i \omega t}$, which is suppressed in the notation. Let $\eta_{u}=p^{\prime}$ and $\eta_{d}=q^{\prime}$, where $p^{\prime}, q^{\prime} \in R$ are arbitrary. Since the problem is linear, we can instead consider:

$$
p:=\frac{\eta_{u}+\eta_{d}}{2}:=\frac{p^{\prime}+q^{\prime}}{2}
$$



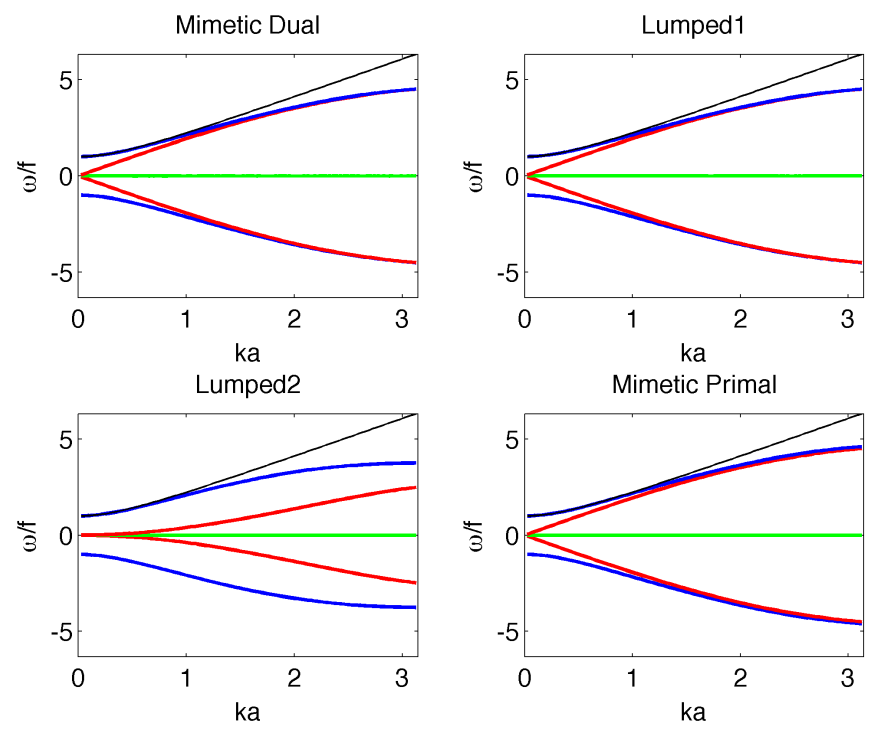

Figure 3: Dispersion curves for the different variants of mimetic discretizations for $a / L_{R}=$ 0.5 (fine mesh).

and

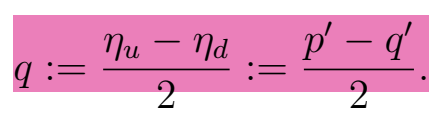

The first one corresponds to the physical modes, and the second one to the spurious numerical modes of triangular C-grid in the limit of small wavenumbers. We consider the case of spurious modes for which the momentum equations are as follows:

$$
\begin{aligned}
& -i \omega u_{a}+C_{a}+3 g q / h=V_{a}, \\
& -i \omega u_{b}+C_{b}+3 g q / h=V_{b}, \\
& -i \omega u_{c}+C_{c}+3 g q / h=V_{a},
\end{aligned}
$$

where $h$ denotes the height of the triangle, $C_{a}=f(\sqrt{3} / 2)\left(u_{b}-u_{c}\right)$ the Coriolis operator, the Coriolis terms at the other edges are given cyclic permutation. The precise form of the Coriolis term is irrelevant, it is only important that $C_{a}+C_{b}+C_{c}=0 . V_{a, b, c}$ are the contributions from viscosity to be specified later. The continuity equation becomes

$$
-i \omega q-H \delta(2 / h)=0, \quad \text { with } \delta:=\left(u_{a}+u_{b}+u_{c}\right)
$$



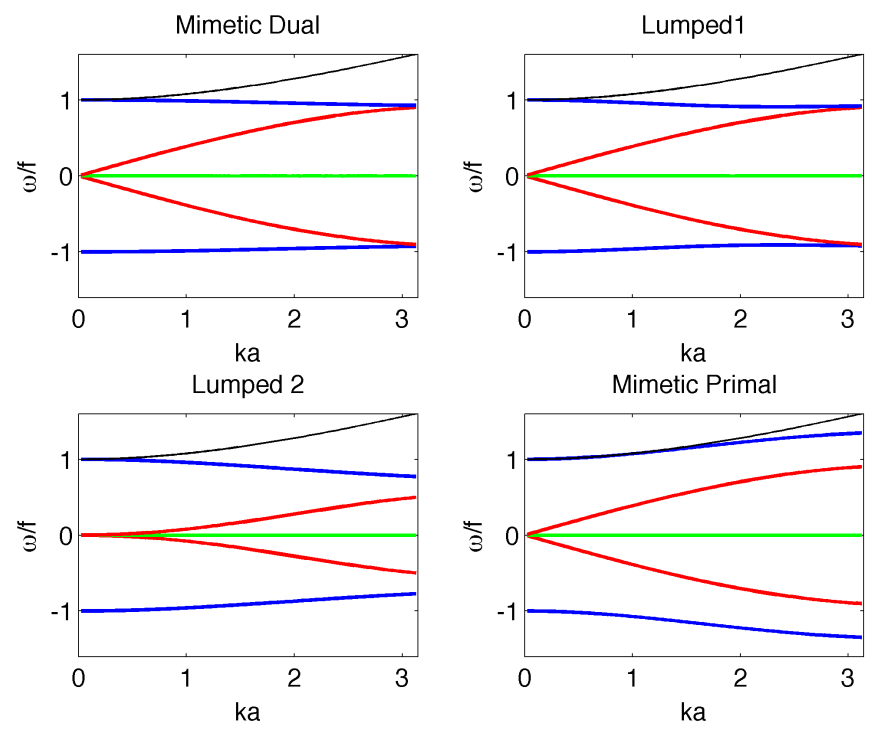

Figure 4: The same as in Fig. 3, but for $a / L_{R}=2.5$ (coarse mesh).

on $u$-triangles and the equation on $d$ triangles is just identical to this one. If viscosy is absent, summing momentum equations we get

$$
-i \omega \delta+9 g \frac{q}{h}=0
$$

Eliminating the sum of velocities between this equation and (16), we obtain

$$
\begin{aligned}
& \quad(-i \omega)^{2}(h / 2 H)+9 g / h=0, \\
& \text { or } \omega= \pm 3 \cdot 2^{1 / 2} c / h
\end{aligned}
$$

If $q \neq 0$, then (17) implies that $\delta \neq 0$ and spurious modes follow. From (18)we infer that for high resolution is $(h<<1)$ the frequency of the modes is high, and they are not necessarily excited by slow dynamics. If resolution is about the Rossby radius, their frequency will be about the Coriolis frequency. Consider now the mimetic discretization and for simplicity in the Lumped-1 configuration (14). In this case (16) will be modified. Since in the limit of small wavenumbers $\delta$ is in the null space of $P^{T} P$, (16) becomes

$$
-i \omega q=0
$$

Hence either $\omega$ or $q$ vanish. If we assume $\omega=0$, then from (17) it follows that $q=0$. The same argument allows to conclude that the assumption 
$q=0$ implies $\omega=0$. In this case the momentum equations are satisfied with $u_{a}=u_{b}=u_{c}$, and this is an element of the null-space of $P^{T} P$. The important aspect to note is that in the limit of small wavenumbers the spurious mode is left in horizontal velocities, but not in elevation. Consequential there is no noise in elevation or -in case of an ocean model- in the isopycnal displacements.

This analysis prompts the question of how to dump the mode in velocity. An obvious suggestion is to model the viscous operator as

$$
V(\mathbf{u})=\nu_{2} \nabla \nabla \cdot \mathbf{u}+\nu_{1}(\nabla \nabla \cdot \mathbf{u}-\nabla \times \nabla \times \mathbf{u})
$$

where one would try to find a trafe-off between the parameters $\nu_{1}, \nu_{2}$. High values of $\nu_{2}$ would have little impact on the well-resolved physical waves that but it will strongly damp the for spurious waves, especially in the limit of small wavenumbers. Indeed, it is easy to see, considering only the case $\nu_{1}=0$, that

$$
V_{a}=V_{b}=V_{c}=-\nu_{2}\left(18 / h^{2}\right) \delta .
$$

Thus the second viscosity penalizes the spurious component in velocities very efficiently (note the appearance of $-18 / h^{2}$ as an eigenvalue of the operator. Taking this in account it is easy to show that spurious mode is purely decaying one in the mimetic case, with $-i \omega=-\nu_{2}\left(18 / h^{2}\right)$. For C-grid, using the perturbation method, we find that the decrement will one half of that for mimetic. Numerical computations support this analysis and show that in the mimetic case the spurious mode frequency remains purely imaginary over some interval of wavenumbers.

\subsection{Kelvin and Rossby waves}

Rossby waves occur only because the Coriolis parameter varies, and we will take a beta-plane approximation here. While exploring the Poincaré waves was relatively straightforward, the analysis of Rossby waves can only be carried out numerically. Deriving the discrete potential vorticity equation from the discretized shallow water equation does not lead to simplifications if the Coriolis parameter is variable. The Kelvin waves can be explored on an f-plane. Although the analysis of Kelvin waves could have been done analytically, it will be provided here as a by-product of the numerical approach to follow. 


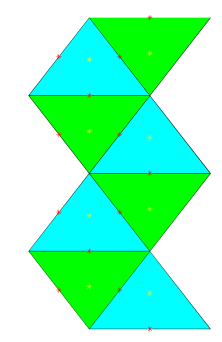

Figure 5: Schematics of channel mesh.

\section{Rossby Waves}

Consider a mesh that is zonally re-entrant, and is composed by $N$ bands of pairs of upward and downward pointing triangles. The basic block is shown in Fig. 5 for $N=4$, it is assumed to be repeating infinitely in the zonal direction. The $x$-coordinate dependence is taken as $e^{i k x}$ and everywhere further the length is made dimensionless with the triangle side $a$. The dimensionless channel width is $L=N h=N \sqrt{3} / 2$. In this arrangement the state vector contains $N+1$ degrees of freedom in $u_{a}(n), n=1, \ldots, N+1$, with $u_{a}(1)=u_{a}(N+1)=0$, because of impermeability at the channel boundary, and $N$ degrees of freedom in each of other quantities $\left(u_{b}, u_{c}, \eta_{u}, \eta_{d}\right)$, leading to $5 N+1$ degrees of freedom in total. The calculation of the corresponding system stiffness matrices can be done analogously to the previous section 3.1, the details can be found in Appendix 4.

Our goal now is to compute the eigenvalues of the matrices $M_{C}, M_{\text {Primal }}, M_{\text {Dual }}, M_{L_{1}}$ and $M_{L_{2}}$ (cf. eqs. (11)-(15)) as a function of the wavenumber $k$. They will contain the Rossby branches, two Kelvin branches and the branches of Poincaré and spurious modes.

In dimensionless variables (the frequency scaled with $f_{0}$ ), the dispersion relation for the Kelvin waves on an $f$-plane is

$$
\omega=k / r
$$

for the wave with the coast to the right in the Northern Hemisphere. The Rossby wave dispersion relation on the beta-plane (and in QG scaling) is

$$
\omega=-\left(\beta a / f_{0}\right) \frac{k}{k^{2}+l^{2}+r^{-2}} .
$$


The eigenvalue problem is solved for a discrete set of zonal wavenumbers $k$ in order to determine the meridional eigenmodes and their associated eigenfrequencies. One expects that the modes will be approximately characterized by the meridional wavenumber $l$ being multiple of $\pi / L=2 \pi /\left(N 3^{1 / 2}\right)$. The results shown below are obtained with the number of triangular bands in the zonal direction is $N=30$. Increasing $N$ further will widen the channel, the meridional modes will change too, but no qualitative changes will ensue as compared to the pattern presented.

Figures 6 and 7 present the dispersion curves for the standard C-grid and the Mimetic-Dual respectively computed for the fine mesh $r=0.5$. The bottom panels in both cases correspond to the upper panels, but zoomed in the vicinity of the $k$ axis, to visualize the Rossby wave frequencies which are too small to be presented simultaneously with the other waves. The black lines are the theoretical estimates for the Kelvin wave (upper panel) and the lowest-order beta-plane Rossby wave (bottom panel). The case of C-grid, as expected, gives a rather accurate estimate of the Kelvin wave dispersion, and reproduces families of Poincaré and spurious (high-frequency) modes discussed in the previous section (top panel). The representation of Rossby waves is rather accurate too at low wavenumbers, but deviations increase as $k$ is increased. Partly this is linked to the fact that the Rossby radius is only marginally resolved. Also note that the black theoretical curve does not present the true solution of the eigenvalue problem for the channel. However, we see that the highest meridional modes (the color is shifting to green) have a wrong frequency sign (wrong phase speed direction). Increasing $N$ does not eliminate this feature, nor does the reduction of $r$.

The lowest-order branches of Rossby waves in the Mimetic-Dual case show a larger error compared to the $\mathrm{C}$-grid case. The cause for that proves to be the use of the Coriolis operator $C_{M}$. If it is replaced by $C$ (mimetic-primal, not shown), the behaviour of the Rossby waves of standard C-grid is recovered. We thus conclude that the behaviour of Rossby waves is insensitive to the presence of divergence averaging (through $P^{T} P$ ), but is related to the locality of the Coriolis operator. The operator $C_{M}$ possesses a larger stencil, hence excessive averaging and reduced accuracy.

Rendering the Kelvin waves presents a more delicate issue. Formally, in Mimetic-Dual (see Fig. 7) there is the Kelvin mode starting from zero frequency. However, low-frequency spurious modes come close to it, their number increasing with $N$ increased. It is therefore possible that they will be excited together with the low-frequency Kelvin-wave signals in solutions of 
initial-value problems.

We do not show other cases. The case Lumped-1, despite the inconsistency between the divergence and gradient operator, leads to solutions which are rather close to the Mimetic-Dual. Lumped-2, in contrast, fails to reproduce the Kelvin wave as a distinct branch.
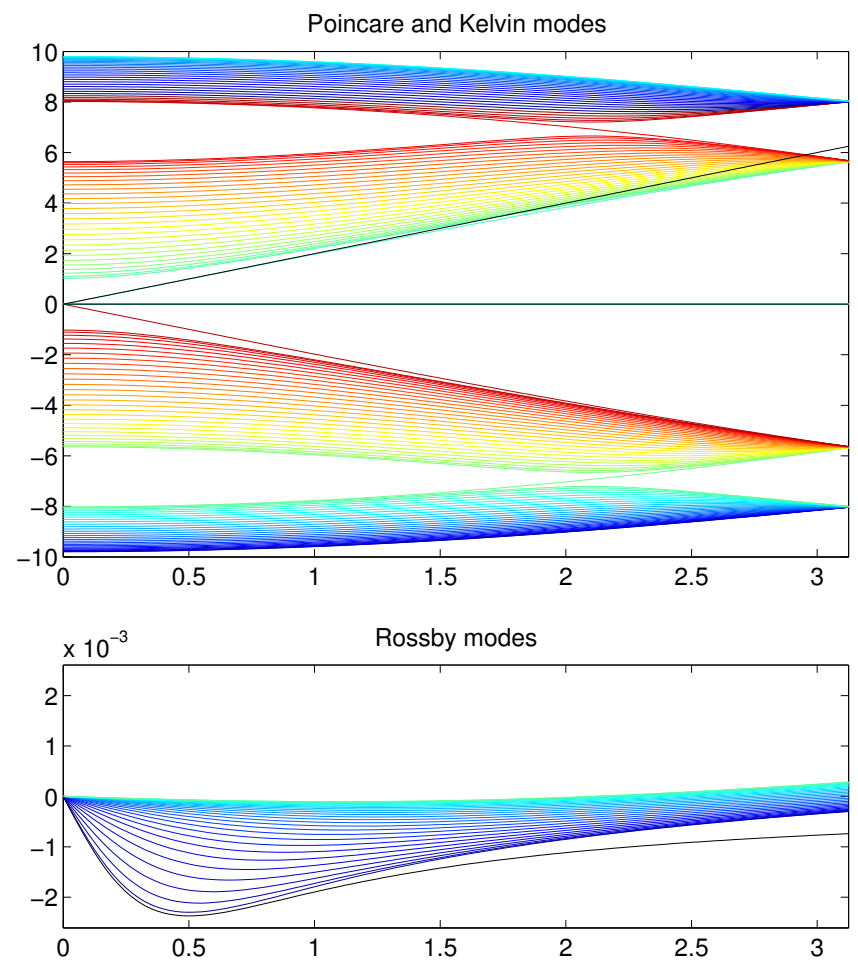

Figure 6: Poincaré, Kelvin (top) and Rossby (bottom) waves in case 1 (C-grid) for $r=$ $a / L_{R}=0.5$. The black lines are the theoretical dependencies (see the text). The numerical Kelvin wave is almost indistinguishable from the theoretical one at small $k a$. Colorbar is cycled to show frequencies in an ascending order.

\section{Kelvin Waves}

The purpose of this section is to illustrate that Kelvin wave propagation is maintained by the mimetic discretization. We simulate a Kelvin wave in a spherical basin, in which it is initiated with an exponential perturbation of the initial fluid thickness that takes its maximum next to the boundary. We 

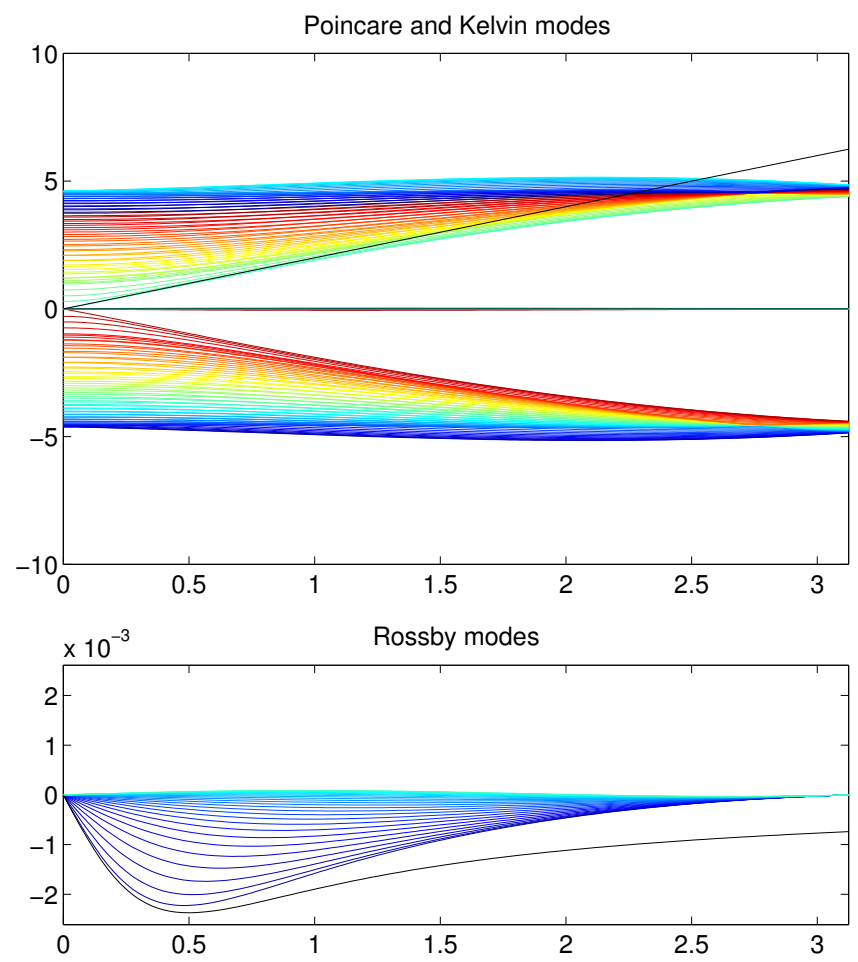

Figure 7: The same as in Fig. 6, but for case 2 (mimetic discretization). There are (spurious) modes with frequencies lower that the Coriolis frequency which will be excited together with the Kelvin wave.

then study the behaviour of the Kelvin wave along the basin boundary. We use the model configuration lumped-1, described in (9).

The parameters of the experiment are as follows. The basin has an extent from $3^{\circ}$ to $18^{\circ}$ longitude and from $40^{\circ}$ to $48^{\circ}$ latitude, and consists of 1430 triangles with edge lenght of $30 \mathrm{~km}$ (see Fig. 8). The circumference of the basin is $\approx 3360 \mathrm{~km}$. In order to avoid to excite Rossby waves we assume an f-plane with the Coriolis parameter $f \approx 2 \times 10^{-5}$, which corresponds to the Coriolis force at a latitude of $8^{\circ}$. The depth of the fluid is $\mathrm{H}=5 \mathrm{~m}$, we use a reduced gravity $g:=9.80665 / 10$. These parameters result in a wave speed $c=\sqrt{g H} \approx 2.2 \frac{m}{s}$ and a Rossby radius of $R=\sqrt{g H} / f \approx 109 \mathrm{~km}$. The resolution of $30 \mathrm{~km}$ resolves the Rossby radius. The initial condition is a local exponential perturbation of $H$ near the southern basin boundary and is the form

$$
\eta_{0}(x, y):=H+e^{-r(x, y)}
$$


with $r$ the distance of $(x, y)$ to a fixed point at middle of the southern boundary of the basin. The experiment is integrated over one year with a time step of $600 \mathrm{~s}$. With these parameters a Kelvin wave needs $\approx 18$ days to travel along the boundary around the basin.

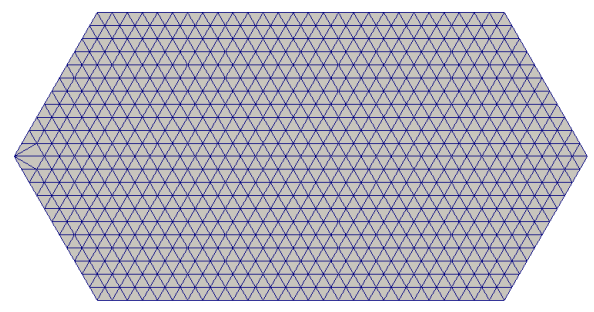

Figure 8: Grid for the Kelvin wave experiment. The outer layer of triangle shows the boundary.

Figure 9 shows the wave initially, after 6 month and after one year. It can be seen that the wave propagates parallel to the boundary.

In Fig 10 the time series of wave elevation of an individual triangle at the location of the initial perturbation is shown. The temporal distance between two peaks is approximately 18 days, which corresponds to the estimate based on the wave speed and the basin circumference.

Fig 11 illustrates another Kelvin wave characteristic, the change in amplitude at a specific location, here the position of the initial perturbation, in dependence of the distance to the domain boundary. The initial width broadens with time.

This example shows that if initial conditions have negligible projection on spurious modes, the dynamics of Kelvin wave is reproduced by the mimetic discretization. 


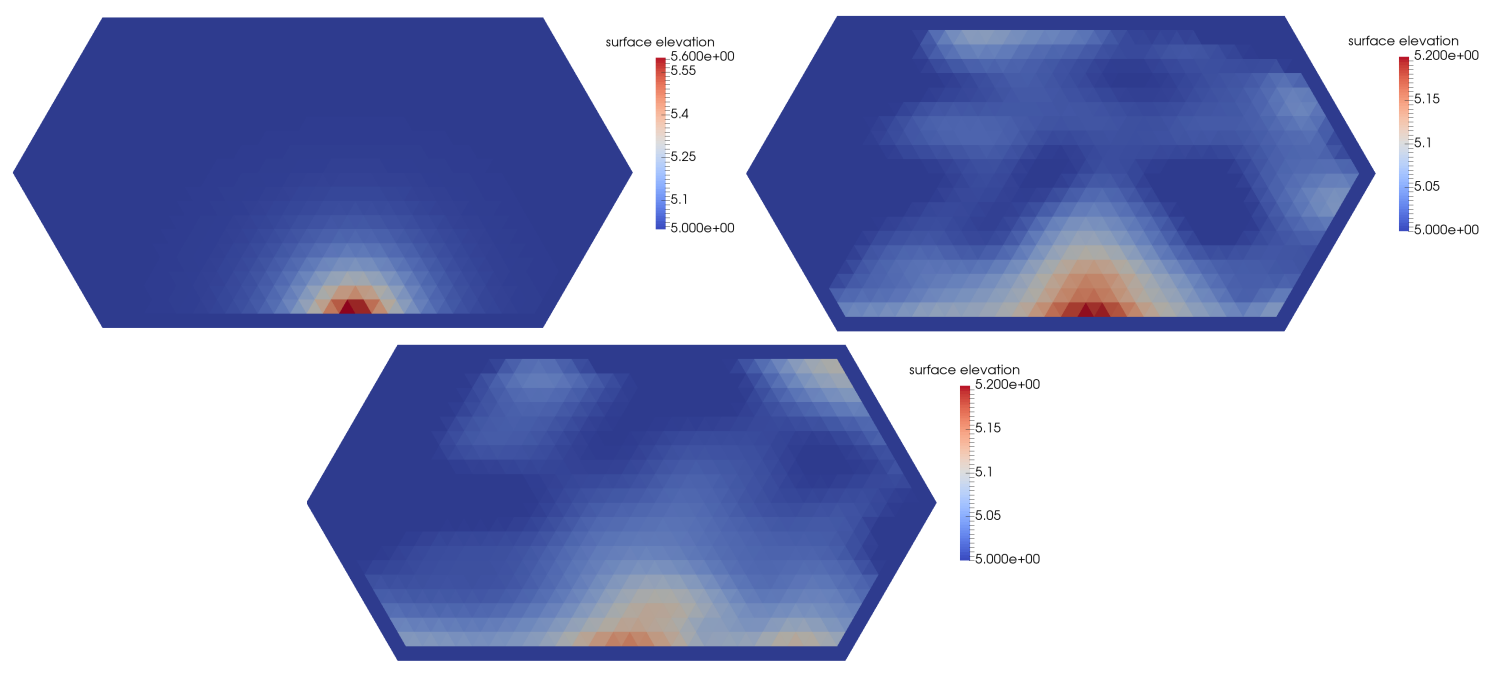

Figure 9: Kelvin wave at initial time (top left) after 6 month (top right) and after one year (lower figure). Please note the change of the color scale.

\section{Conclusions}

We present the analysis of dispersion properties of linearized shallow water equations, discretized on a triangular mesh with the mimetic approach [7], and its several simplified forms. While the mimetic approach employs the variable placement of the standard triangular $\mathrm{C}$-grid, it relies on a different scalar product. As a consequence, it operates with averaged velocities $P^{T} P v_{e}$ in the continuity equation. This averaging leads to essential improvements against the behaviour of triangular C-grids which develop a checkerboard pattern in the divergence of horizontal velocity. The noisy pattern of triangular C-grids is the consequence of spurious modes maintained by this discretization. Their origin is of purely geometrical nature and is linked to the excessive number of pressure points compared to the velocity points. We show that the mimetic discretization does not eliminate spurious modes, but reduces their frequency at small wavenumbers and, hence, their manifestations (the divergence is proportional to the amplitude of surface displacement and frequency).

Although spurious modes of mimetic discretization become low-frequency at small wavenumbers, they are still detached from the Rossby wave dynamics. However, there are low-frequency spurious modes in the mimetic case which 


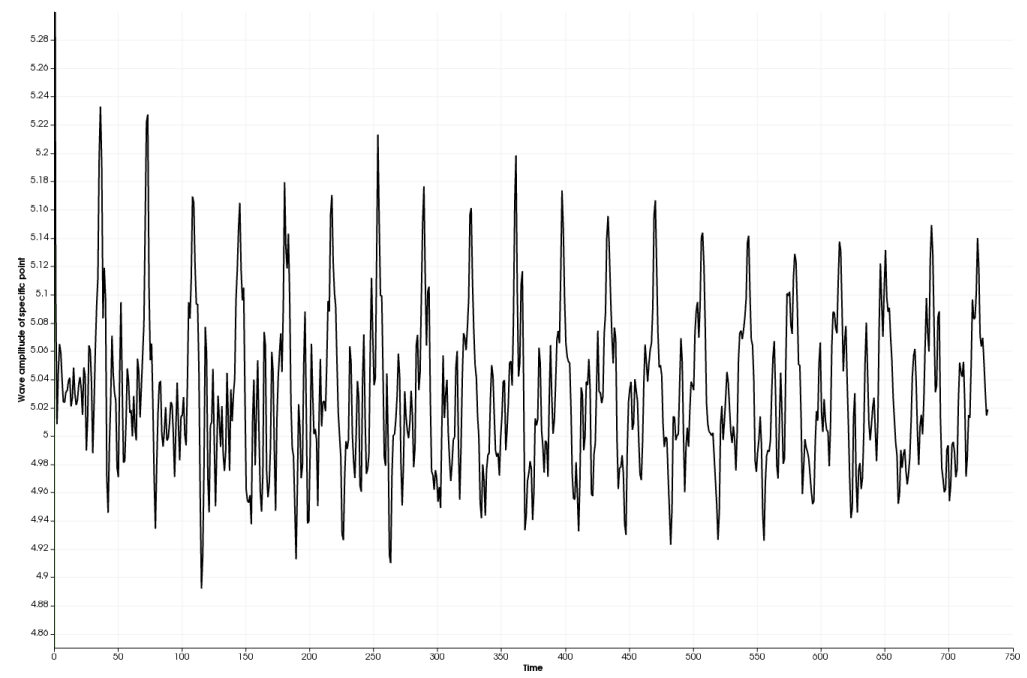

Figure 10: Wave amplitude of an individual triangular cell as a time series. The cell is located at the position of the initial perturbation. The labels of the $\mathrm{x}$-axis are the numbers of the 12-hourly output over the 12 month of integration.

lie close to the Kelvin wave branch. Learning about all implications of this behaviour is reserved for future.

The mimetic approach introduces a mass matrix $\left(P^{T} P\right)$ with the velocity time derivative, which increases the computational cost of this discretization. We show that lumping done simultaneously for the velocity derivative and pressure gradient (Lumping-1) does not lead to any visible artefacts compared to full mimetic discretization. The other scheme (Lumping-2) with lumping only for the time derivative, shows a reduced accuracy, but its spurious modes preserve low frequency for a broader interval of wavenumbers. There is also sensitivity to the form of the Coriolis operator. The form $C=P^{T} f \mathbf{k} \times P$ leads to more accurate dispersion relation on coarse meshes and, additionally, keeps the correct sigh of group velocity in this case.

To conclude, we would like to emphasize the following points.

- The presence of the averaging operator $P^{T} P$ in the mimetic approach implies that the velocity field $P^{T} P v_{e}$, and not $v_{e}$, satisfies the continuity equation. Same as any averaging operator, this one reduces the accuracy of the discrete dispersion for physical modes compared to the standard C-grids, but this reduction is very moderate and the accuracy 

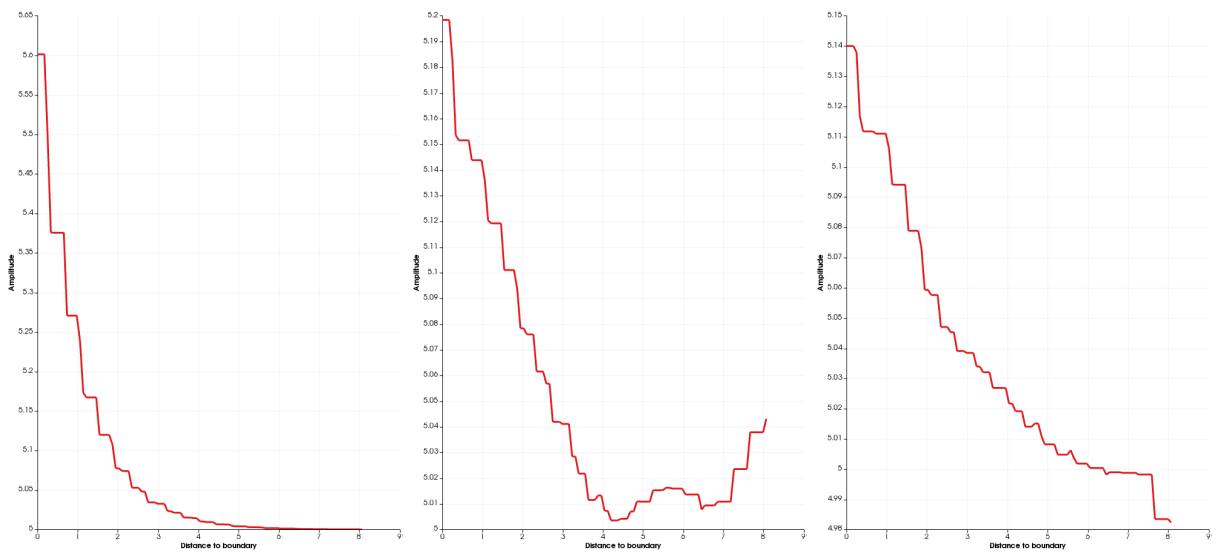

Figure 11: Change in wave amplitude with increasing distance to the boundary at the intial datum (left), after 6 month (middle) and after 12 month (right). The specific location is the position of the intial perturbation. The $\mathrm{x}$-axis gives the distance to the southern boundary in degrees.

remains high, as expected from a discretization of the C-grid type. The choice of the Perot reconstructions for implementing $P^{T} T P$ is not essential, it was motivated by their simplicity that makes them amenable for the dispersion analysis carried out here. The impact of different reconstructions within the operator products $P^{T} P$ and $\hat{P}^{\dagger} \hat{P}$ on dispersion relations as well as on the model solution in general remains to be investigated.

- The divergence operator acting on the velocity field $P^{T} P v_{e}$ presents the generalization of the divergence averaging discussed by [17] to nonuniform meshes. Thus, the mimetic approach offers a way to implement this averaging without violating energetic consistency.

- Despite the reduced frequency of spurious modes at small wavenumbers, their frequencies become close to those of physical modes for high wavenumbers, $k a>1$, see Fig. 3 and 4 . In the case of 3D primitive equations the implication is that in eddying flows, characterized by the direct cascade of enstrophy to small scales, or for forcing on superinertial frequencies noise may still be provoked, and additional measures might be required to eliminate excessively small scales (high frequencies) in the velocity and relative vorticity. This is the subject of future 
research.

\section{Appendix: Matrices for Dispersion Analysis of Kelvin and Rossby Waves}

The expressions for the operators required in sect. 3.2 can be derived, by introducing the phase factor $\delta=e^{i k / 4}$ and using index $n$ to account for

the difference in the meridional direction instead of the meridional phase factor of section 3.1. Thus, the matrix of the divergence operator on upward triangles becomes

$$
\begin{aligned}
& D_{u}(n, n)=-2 / h, \\
& D_{u}(n, N+1+n)=-2 \delta / h, \\
& D_{u}(n, 2 N+1+n)=-2 \delta^{*} / h,
\end{aligned}
$$

with $\delta=e^{i k / 4}$ and on downward triangles we obtain

$$
\begin{aligned}
& D_{d}(n, n+1)=2 / h, \\
& D_{d}(n, N+1+n)=2 \delta^{*} / h, \\
& D_{d}(n, 2 N+1+n)=2 \delta / h,
\end{aligned}
$$

and non-zero entries of all other operators can be written in the same easy way. For the standard C-grid the problem stiffness matrix becomes

$$
M_{C}=\left(\begin{array}{cc}
C & g G \\
H D & 0
\end{array}\right)
$$

where the Coriolis matrix $C$ is now a $3 N+1$ square matrix, $G$ is $(3 N+1) \times 2 N$ matrix, $D$ is $2 N \times(3 N+1)$ matrix and 0 is the matrix filled with zeros. The Coriolis parameter $f=f_{0}+\beta y$ (with $f_{0}$ be the value at the center, and $\beta$ the meridional gradient) is estimated at cell centers. The expression for the Coriolis operator becomes

$$
C=\left[P_{a} ; P_{b} ; P_{c}\right]\left[-\operatorname{diag}\left(f_{u}\right) P_{u y} ; \operatorname{diag}\left(f_{u}\right) P_{u x} ;-\operatorname{diag}\left(f_{d}\right) P_{d y} ; \operatorname{diag}\left(f_{d}\right) P_{d x}\right] .
$$

Here $f_{u}$ and $f_{d}$ are the vectors consisting of the values of the Coriolis parameter at $u$ and $d$ points respectively (circumcenters of respective cells), and semicolon implies combining row-wise. In this expression, $P_{u x}, P_{u y}, P_{d x}$ and $P_{d y}$ are the components of the forward Perot operator, acting on the velocity 
state vector $\left(u_{a}, u_{b}, u_{c}\right)^{T}$ and returning the components of the velocity vector at $u$ and $d$ locations. Accordingly, $P_{a}, P_{b}, P_{c}$ are the components of the transpose Perot operator acting on $4 N$ vector $\left(u_{u}, v_{u}, u_{d}, v_{d}\right)^{T}$ to get the edge velocities.

For the mimetic dual scheme the Coriolis operator $C_{M}$ is obtained as

$$
C_{M}=\left[\hat{P}_{a}, \hat{P}_{b} ; \hat{P}_{c}\right]\left[\operatorname{diag}\left(f_{v}\right) \hat{P}_{x} ; \operatorname{diag}\left(f_{v}\right) \hat{P}_{y}\right],
$$

here the vector with the values of the Coriolis parameter $f_{v}$ is estimated at triangle vertices. The operators $\hat{P}_{x}$ and $\hat{P}_{y}$ are the components of Perot vertex reconstruction, and the remaining operators implement transpose operation of reconstruction from vertices to edges.

The expression for the stiffness matrices in other cases are obtained similarly to the C-grid case, and similarly to the analysis for the Poincaré waves, with the difference that we are dealing now with the matrices of larger size. The matrix $T$ is now such that $T(1: 3 N+1,1: 3 N+1)=$ $P^{T} P=\left[P_{a} ; P_{b} ; P_{c}\right]\left[P_{u x} ; P_{u y} ; P_{d x} ; P_{d y}\right]$, and its remaining diagonal values are equal to 1 . Once the stiffness matrices are assembled, they are 'compacted' (by removing rows and columns 1 and $N+1$ ), which corresponds to imposing boundary conditions.

\section{Appendix: Mimetic discretization on quads}

The impact of mimetic discretization is much more apparent on quadrilateral meshes than on triangular meshes. The intention of this section is to illustrate it.

On a regular quadrilateral mesh we seek for solutions of the form $(u, v, \eta)=$ $\left(u_{0}, v_{0}, \eta_{0}\right) e^{i K x+i L y-i \omega t}$, with $K$ and $L$ the dimensional wavenumbers. In this case we need only three degrees of freedom. (Any two $u$-velocities are connected through a phase shift, and same for other variables.) In what follows we work with amplitudes and omit the subscript ' 0 '. We introduce the dimensionless wavenumbers

$$
k=K \Delta x / 2, \quad l=L \Delta y / 2,
$$

and set $\Delta x=\Delta y=a$ for simplicity. The Perot reconstruction to cell centers is

$$
\mathbf{u}=\left(P_{x} u, P_{y} v\right), \quad P_{x}=\cos k, \quad P_{y}=\cos l
$$


Here and below we write expressions with respect to the point where they are defined, keeping only relative phases. The full velocity vector $\mathbf{u}$ appearing here is defined at cell centers (at scalar points). The transpose operators are computed similarly, so that

$$
P^{T} P=\left(\begin{array}{cc}
T_{x} & 0 \\
0 & T_{y}
\end{array}\right)
$$

with $T_{x}=(\cos k)^{2}$ and $T_{y}=(\cos l)^{2}$. Since this matrix is diagonal, all computations are elementary.

Full velocity reconstruction to the vertex points is

$$
\mathbf{u}=\left(\hat{P}_{x} u, \hat{P}_{y} v\right), \quad \hat{P}_{x}=\cos l, \quad \hat{P}_{y}=\cos k .
$$

Here it is more convenient to reconstruct in this way, on irregular meshes we have to use the vertex Perot reconstruction instead. Using full velocities at vertices to estimate the Coriolis terms at edges we get the Coriolis operator as

$$
C_{M}=f \cos k \cos l\left(\begin{array}{cc}
0 & -1 \\
1 & 0
\end{array}\right)
$$

Note that the expression for the standard C-grid and Mimetic-Dual Coriolis operator are identical on quads. Indeed, in both cases they are obtained by just averaging over the nearest neighbors. They are, however, different on general (irregular) meshes. The rest is relatively straightforward. We have for the gradient operator

$$
G_{x}=(2 i / a) \sin k, \quad G_{y}=(2 i / a) \sin l, \quad G_{M x}=T_{x} G_{x}, \quad G_{M y}=T_{y} G_{y} .
$$

Similarly, for the divergence we have the row matrix

$$
D=\left(\begin{array}{ll}
D_{x} & D_{y}
\end{array}\right)=(2 i / a)\left(\begin{array}{ll}
\sin k & \sin l
\end{array}\right), \quad D_{M}=\left(\begin{array}{ll}
T_{x} D_{x} & T_{y} D_{y}
\end{array}\right) .
$$

The linearized shallow water equations for the mimetic discretization become

$$
\begin{aligned}
& -i \omega T_{x} u-f v \cos k \cos l+g T_{x} G_{x}=0 \\
& -i \omega T_{y} v+f u \cos k \cos l+g T_{y} G_{y}=0 \\
& -i \omega \eta+H D_{x}\left(T_{x}\right) u+H D_{y}\left(T_{y}\right) v=0 .
\end{aligned}
$$

A suitable choice of the factors $T_{x}$ and $T_{y}$ leads to the different mimetic configurations. The standard C-grid follows on setting $T_{x}=T_{y}=1$. Case 
Lumping- 1 would imply setting $T_{x}=T_{y}=1$ in the momentum equations, and case Lumping-2 doing this only for the time derivative term in the momentum equations. Case Mimetic-Primal is identical to Mimetic-Dual because of the identical Coriolis operators.

Inserting the expression for $u$ and $v$ in the momentum equation above into the height equations implies

$-i \omega+\frac{c^{2}}{a^{2}} \frac{a^{2}}{-\omega^{2} T_{x} T_{y}+\bar{f}^{2}}\left[D_{x}\left(T_{x}\right)\left(i \omega T_{x} T_{y} G_{x}-\bar{f} T_{y} G_{y}\right)+D_{y}\left(T_{y}\right)\left(i \omega T_{x} T_{y} G_{y}+\bar{f} T_{x} G_{x}\right)\right]=0$.

Here $\bar{f}=f \cos k \cos l$. The two terms with $\bar{f}$ inside the square brackets should disappear in order to have a correct geostrophic mode. Together they give

$$
\bar{f}\left[-D_{x}\left(T_{x}\right) T_{y} G_{y}+D_{y}\left(T_{y}\right) T_{x} G_{x}\right] .
$$

This combination is zero on pure C-grid. It is also zero for mimetic discretization and in case Lumping-2. For Lumping-1, however, when lumping involves the gradient operator, there is no cancellation, and this case should be discarded on quads (note that, in contrast, Lumping-1 is a preferred option for triangles). The resultant dispersion equations are

\section{C-grid:}

$$
(\omega / f)^{2}=\cos ^{2} k \cos ^{2} l+4\left(L_{r} / a\right)^{2}\left(\sin ^{2} k+\sin ^{2} l\right)
$$

\section{Mimetic-Dual:}

$$
(\omega / f)^{2}=1+\left(L_{r} / a\right)^{2}\left(\sin ^{2} 2 k+\sin ^{2} 2 l\right),
$$

\section{Lumping-2:}

$$
(\omega / f)^{2}=\cos ^{2} k \cos ^{2} l+\left(L_{r} / a\right)^{2}\left(\sin ^{2} 2 k+\sin ^{2} 2 l\right) \cos ^{2} k \cos l .
$$

Mimetic-Dual does a nice job with the Coriolis, because the 'inversion' of mass matrices coming with time derivative in the momentum equation takes care about removing averaging associated to the Coriolis operator. However, there is also price for that. The accuracy of wave dispersion drops dramatically because of doubling of the argument of sine functions. Lumping- 2 combines the worst sides of both standard C-grid and Mimetic-Dual. So there is no clear winner in this comparison. The mimetic discretization respects 
physics by maintaining $f$ as a cut-off frequency, but the standard C-grid still has much higher accuracy if the Rossby radius is resolved. Besides, the mimetic dispersion becomes similar to the A-grid dispersion in this case.

[1] Arakawa A., Lamb V. R., 1981, A potential enstrophy and energy conserving sheme for the shallow water equations. Mon. Wea. Rev., 109, $18-36$.

[2] Zhang, Y.L., Baptista, A.M., Myers, E.P., 2004. A cross-scale model for 3D baroclinic circulation in estuary-plume-shelf systems: I. Formulation and skill assessment. Cont. Shelf Res. 24, 2187-2214.

[3] Casulli, V., Walters, R. A., 2000. An unstructured grid, threedimensional model based on the shallow water equations. Int. J. Numer. Meth. Fluids., 32, 331-348.

[4] Danilov, S., 2010. On utility of triangular C-grid type discretization for numerical modeling of large-scale ocean flows, Ocean Dynamics 60(6), 1361-1369.

[5] Fringer, O. B., Gerritsen, M., Street, R. L., 2006. An unstructured-grid, finite-volume, nonhydrostatic, parallel coastal ocean simulator. Ocean Modelling 14, 139-173.

[6] Gasmann, A., Inspection of hexagonal and triangular C-grid discretizations of the shallow water equations. J. Comput. Phys. 230, 2706-2721.

[7] Korn, P., Formulation of an Unstructured Grid Model for Global Ocean Dynamics (in review)

[8] Korn, P., Linardakis, L., A Class of Potential Enstrophy and Energy Conserving Discretizations of the Shallow-Water Equations on Unstructured Grids of Variable Resolution (in preparation)

[9] Kleptsova, O., Pietrzak, J. D., Stelling, G. S., 2009. On the accurate and stable reconstruction of tangential velocities in C-grid ocean models. Ocean Modelling, 28, 118-126.

[10] Le Roux, D. Y., Rostand, V., Pouliot, B., 2007. Analysis of numerically induced oscillations in 2D finite-element shallow-water models. Part I: Inertia-gravity waves. SIAM J. Sci. Comput. 29, 331-360. 
[11] Perot, B. 2000. Conservation properties of unstructured staggered mesh schemes. J. Comput. Phys., 159, 58-89.

[12] Raviart, P. A., Thomas J. M., 1977. A mixed finite element method for 2nd order elliptic problems. In Mathematical Aspects of the Finite Element Methods, Galligani I, Magenes E (eds). Lecture Notes in Mathematics. Springer: Berlin, 292-315.

[13] Ringler, T. D., Thuburn, J., Klemp, J. B., Skamarock, W. C., 2010. A unified approach to energy conservation and potential vorticity dynamics for arbitrarily-structured C-grids. J. Comput. Phys. 229, 3065-3090.

[14] Rostand, V., Le Roux, D. Y., Carey, G., 2008. Kernel analysis of the discretized finite difference and finite element shallow-water models. SIAM J. Sci. Comput. 31, 531-556.

[15] Stuhne, G. R., Peltier, W. R., 2006. A robust unstructured grid discretization for 3-dimensional hydrostatic flows in spherical geometry: A new numerical structure for ocean general circulation modeling. J. Comput. Phys. 213, 704-729.

[16] Walters, R. A., Hanert, E., Pietrzak, J., Le Roux, D. Y., 2009: Comparison of unstructured, staggered grid methods for the shallow water equations, Ocean Modelling, 28, 106-117.

[17] Wan, H., Giorgetta, M. A., Zängl, G., Restelli, M., Majewski, D., Bonaventura, L., Fröhlich, K., Reinert, D., Rípodas, P., Kornblueh, L., Förstner, J., 2013. The ICON-1.2 hydrostatic atmospheric dynamical core on triangular grids - Part 1: Formulation and performance of the baseline version Geosci. Model Dev., 6, 735-763.

[18] Wolfram, P. J., Fringer, O. B., 2013. Mitigating horizontal divergence "checker-board" oscillations on unstructured triangular C-grids for nonlinear hydrostatic and nonhydrostatic flows. Ocean Modelling, 69, 64-78.

[19] G. Zängl, D. Reinert, P. Ripodas, M. Baldauf, The ICON (ICOsahedral Non-hydrostatic) modelling framework of DWD and MPI-M: Description of the non-hydrostatic dynamical core, Q. J. R. Meteorol. Soc. 141, (2015), 563-579 